\title{
MÉTODO DE AVALIAÇÃO DE EQUIPAMENTOS PARA DIRECIONAMENTO DE VEÍCULOS AGRÍCOLAS E EFEITO DE SINAIS DE GNSS
}

\author{
JOSÉ P. MOLIN ${ }^{1}$, FABRICIO P. POVH², VANDERSON R. DE PAULA ${ }^{3}$, JOSÉ V. SALVI ${ }^{4}$
}

RESUMO: Pesquisas indicam que o uso de novas técnicas no campo da mecanização agrícola, como o tráfego controlado por meio de direcionamento com auxílio de Sistemas de Navegação Global por Satélites (GNSS), pode diminuir as exigências por energia associadas às operações de campo e aumentar a produtividade da mão de obra e da cultura. Alguns autores já utilizaram diferentes metodologias para avaliar erros de paralelismo em sistemas de orientação de veículos, porém não há um procedimento-padrão para medi-los. Sendo assim, o objetivo deste trabalho foi implementar uma planilha eletrônica específica, determinar e comparar a acurácia no paralelismo de dois sistemas distintos de orientação de veículos (uma barra de luz e um piloto automático com atuador de volante) em trajetórias retilíneas e utilizando sinal de GPS com dois sistemas de correção diferencial (um com algoritmo interno e outro, um sinal via satélite com acurácia submétrica). Para a avaliação dos sistemas de correção, utilizou-se como referência o sinal de correção diferencial RTK (Real Time Kinematic) para realização de cinco passadas paralelas sucessivas para cada sistema de correção, caracterizando cinco repetições de cada tratamento. A planilha viabilizou o método de avaliação utilizado e os erros apresentados para as correções nos dois equipamentos analisados mostraram-se compatíveis com operações que exijam acurácia de paralelismo da ordem de decímetros.

PALAVRAS-CHAVE: sistemas de orientação, piloto automático, GPS.

\section{METHOD FOR EVALUATION OF GUIDANCE EQUIPMENTS FOR AGRICULTURAL VEHICLES AND GNSS SIGNALS EFFECT}

\begin{abstract}
Researches indicate that the use of new techniques in agricultural machinery, as controlled traffic by steering systems with the use of Global Navigation Satellite Systems (GNSS) may decrease the energy demand associated to field operations and increase labor efficiency and crop yield. Some authors already use different methodologies to evaluate the parallelism errors in guidance systems for vehicles, but there is no standard methodology to measure them. So, the objective of these work was to develop a specific spreadsheet, determinate and compare the accuracy in the parallelism of two distinct guidance systems (a light bar and an universal autopilot) working at straight path using GPS signal with two differential correction systems (one with internal algorithm and the other with satellite signal and sub metric accuracy). To evaluate both systems a RTK (Real Time Kinematic) differential correction was used, realizing five parallel and successive paths for each system, characterizing five replications for each treatment. The spreadsheet enabled the evaluation method used, and the errors for both equipments and signals analyzed, showed to be compatible with field operations that demand parallelism accuracy in the order of decimeters.
\end{abstract}

KEYWORDS: orientation systems, auto pilot, GPS.

\footnotetext{
${ }^{1}$ Professor Associado, Departamento de Engenharia de Biossistemas, ESALQ/USP, Piracicaba - SP, jpmolin@ usp.br.

${ }^{2}$ Doutorando em Fitotecnia, ESALQ/USP, fppovh@gmail.com.

${ }^{3}$ Eng ${ }^{\mathrm{o}}$ Agrícola, M.Sc., vrpvants@yahoo.com.br.

${ }^{4}$ Doutorando em Fitotecnia, ESALQ/USP, jvsalvi@esalq.usp.br.

Recebido pelo Conselho Editorial em: 28-9-2009
}

Aprovado pelo Conselho Editorial em: 19-12-2010 


\section{INTRODUÇÃO}

A constante tecnificação dos meios de produção vem interferindo no setor agrícola com grande frequência, principalmente pelo tráfego intenso de máquinas nas lavouras. HADAS (1987) constataram que o tráfego de máquinas pesadas no solo proporciona a redução na infiltração de água, condutividade hidráulica, porosidade e aeração, e um consequente aumento de sua densidade e da resistência oferecida ao desenvolvimento dos sistemas radiculares, resultando em baixa produtividade agrícola nesses solos compactados. Uma técnica para mitigar tais problemas é o controle de tráfego com o posicionamento acurado e precisamente paralelo das máquinas agrícolas trafegando nas lavouras.

Experimentos com o uso do tráfego controlado feitos em lavouras de grãos por ACIAR (1998), na Austrália, resultaram em solos com menor resistência ao desenvolvimento do sistema radicular e maior infiltração de água em comparação ao tráfego normal, com um consequente aumento na produtividade, que no caso dos grãos, foi $16 \%$ maior que o manejo convencional. Com relação à redução de danos causados às culturas pelo tráfego de máquinas, ABIDINE et al. (2002) realizaram um experimento em uma lavoura de tomates e constataram que uma redução de $2,5 \mathrm{~cm}$ na distância entre o órgão ativo de um implemento e a linha da cultura implicaram um dano de $4 \%$ nas plantas de tomate.

No que tange ao tráfego controlado, além do ajuste de bitolas e padronização de espaçamentos, são necessárias algumas ferramentas que garantam que os rodados trafeguem na área destinada ao tráfego, contribuindo para operações perfeitamente paralelas e que não dependam exclusivamente da habilidade do operador. $\mathrm{O}$ equipamento conhecido como barra de luzes foi o primeiro a incorporar o direcionamento de veículos agrícolas com sinal GNSS (BAIO \& ANTUNIASSI, 2008). Consiste em um conjunto de sinais luminosos dispostos à frente do operador da máquina ou veículo, ligado a um processador que recebe a informação de posicionamento de um receptor GNSS. Contudo, a tecnologia evoluiu para a automatização da barra de luzes, surgindo os sistemas onde o direcionamento do veículo é feito por um motor auxiliar ligado à direção ou por ação direta no sistema hidrostático de direcionamento, governado pela informação de trajetória gerada por um receptor GNSS.

Para os fabricantes de máquinas agrícolas, a rápida adoção dos sistemas automáticos de orientação nos anos recentes, apesar do custo de implantação, pode ser explicada por vários fatores, como aumento da produtividade no campo, da capacidade operacional, especialmente em condições de baixa visibilidade, redução do uso de insumos e diminuição da fadiga do operador, o que garantiria um retorno rápido do investimento (GAN-MOR \& CLARK, 2001; SILVA et al., 2007). Entretanto, o nível de sofisticação desta tecnologia, que está diretamente relacionado ao custo do equipamento, não precisa necessariamente ser o mesmo em todos os ramos da agricultura, e também não é o mesmo em todos os modelos e marcas de sistemas de orientação existentes no mercado. Assim, deve-se qualificar tais equipamentos e para isso é necessário medir o erro de paralelismo desses sistemas e conhecer o nível de desempenho oferecido, no intuito de melhor adequar o equipamento às necessidades do usuário, contribuindo para um uso racional da tecnologia, com otimização da relação entre custo e benefício.

Alguns autores já utilizaram diferentes metodologias para medir esses erros de paralelismo (MOLIN et al., 2002; ANTUNIASSI \& BAIO, 2004; EHSANI et al., 2002; GAN-MOR et al., 2007) e inicialmente fizeram uso de marcas físicas que pudessem registrar os desvios de paralelismo ao longo da trajetória, porém para condições reais de um tráfego agrícola tais métodos tornaram-se complexos e passíveis de erro. Posteriormente, passou-se a fazer o registro de coordenadas ao longo do trajeto, tendo como referência um GNSS com sistema de correção RTK (Real Time Kinematic), com maior acurácia que o sinal do sistema sendo avaliado. Contudo, as duas técnicas apenas geram os dados em coordenadas, que precisam ser convertidos em informação de erro de paralelismo. 
Assim, devido à necessidade de mensuração desses erros e a ausência de recursos mais aprimorados para fazê-lo, o objetivo deste trabalho foi desenvolver um procedimento para análise de dados de desvios de paralelismo a partir de uma planilha eletrônica específica. Para tanto, foi também determinada e comparada a acurácia no paralelismo de dois sistemas distintos de orientação de veículos em trajetórias retilíneas utilizando sinal GNSS com dois sistemas de correção diferencial.

\section{MATERIAL E MÉTODOS}

Foi planejado e conduzido um experimento para a coleta de dados. Os ensaios foram realizados entre os dias 5 e 7 de novembro de 2008, em uma área de cultivo da Escola Superior de Agricultura "Luiz de Queiroz" da Universidade de São Paulo, em Piracicaba - SP, em torno das coordenadas geográficas $22^{\circ} 42^{\prime} 48^{\prime}$ 'S e $47^{\circ} 36^{\prime} 43^{\prime} \mathrm{W}$. A área utilizada para os ensaios foi uma superfície coberta por resteva de silagem, localizada em uma região livre de obstruções que pudessem interferir no sinal GNSS.

Os equipamentos avaliados foram uma barra de luz e um sistema de direcionamento de veículos automáticos com atuador de motor elétrico, de uso universal, que é conectado ao volante do veículo e que possui recurso para compensar a inclinação de terreno. Ambos os equipamentos foram instalados em um mesmo trator, marca Massey Fergunson, modelo MF 283, e receberam dois tipos de sinal GNSS, um com uso de algoritmo interno de filtragem de erros, largamente utilizado para essas aplicações no mercado brasileiro e outro, um sinal com correção diferencial SBAS ("satellite-based augmentation system", ou via satélite) VBS (OmniStar, Houston, TX, EUA) com acurácia submétrica.

Para a avaliação dos equipamentos e sinais, utilizaram-se como referência as coordenadas geográficas fornecidas por um sistema com sinal de correção diferencial RTK (Real Time Kinematic), uma vez que este tipo de correção é suficientemente acurado para servir na mensuração de erros de alinhamento. Para o fornecimento do sinal de referência, uma antena GPS RTK Trimble 5800 (Trimble Navigation Limited, Sunnyvale, CA, EUA) foi posicionada em alinhamento com a antena geradora do sinal de navegação, instalada sobre o teto do veículo.

O equipamento de referência é composto por um receptor GPS e um receptor de rádio UHF que são instalados no trator e um receptor GPS estacionário, instalado numa base fixa, entre 50 e $200 \mathrm{~m}$ do trator, juntamente com um transmissor de rádio UHF. A base fixa tem a função de gerar e transmitir dados de correção em tempo real para a unidade móvel.

A antena receptora dos sinais GPS avaliada foi fixada no teto do trator, seguindo as orientações do fabricante quanto a distância até o centro do eixo traseiro, distância à antena do GPS RTK e altura em relação ao solo.

A coleta dos dados para a análise foi procedida inicialmente adquirindo um segmento de reta virtual "A-B", utilizando o sinal RTK, sendo esse transmitido aos monitores dos equipamentos, tornando-se a referência das retas paralelas que foram utilizadas para realização de cinco passadas paralelas sucessivas para cada sistema de correção, caracterizando cinco repetições de cada tratamento.

Enquanto o direcionamento do veículo era governado pela barra de luz ou pelo sistema automático de direcionamento, o registro das coordenadas do trajeto era feito a partir do posicionamento dado pelo receptor GNSS RTK. As coordenadas dos trajetos foram consideradas como as verdadeiras trajetórias e comparadas com as coordenadas das retas paralelas à reta "A-B" (Figura 1). 


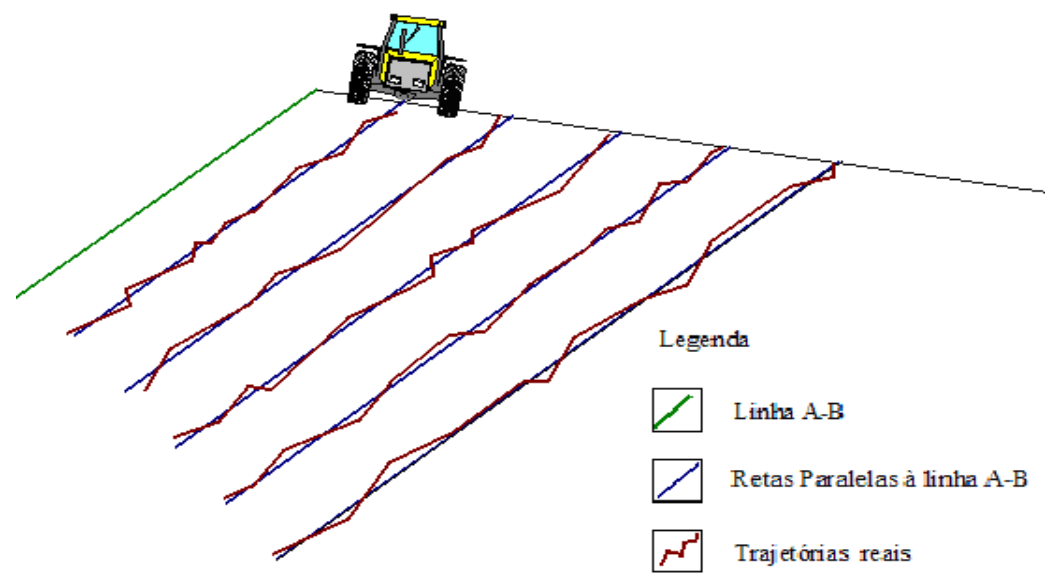

FIGURA 1. Representação dos componentes dos percursos utilizados no ensaio: linha de referência $\mathrm{A}-\mathrm{B}$; retas paralelas à linha $\mathrm{A}-\mathrm{B}$; e trajetórias reais. Representation of the track components used on the tests: reference line A-B; parallel line to A-B; and real tracks.

Para cada tratamento, foi gerado um arquivo, sendo a coleta configurada à taxa de aquisição de uma posição por segundo $(1 \mathrm{~Hz})$ e iniciada no começo de cada tratamento, ainda com o veículo parado. $\mathrm{O}$ veículo percorreu o percurso de cinco passadas paralelas, com velocidade constante, buscando manter uma linha reta paralela à reta "A-B", orientado ou guiado pelo equipamento avaliado. Para gravar as coordenadas coletadas, utilizou-se um computador de mão equipado com o software dedicado (Farm Site Mate ${ }^{\circledR}$ ), ligado diretamente nos monitores da barra de luz e do sistema automático de orientação.

Posteriormente, os dados foram tabulados com o auxílio da planilha eletrônica inicialmente proposta por POVH et al. (2007) no software Microsoft Excel®, e as rotinas de programação foram feitas em Visual Basic ${ }^{\circ}$ na forma de macros. A lógica do processo é apresentada na Figura 2.

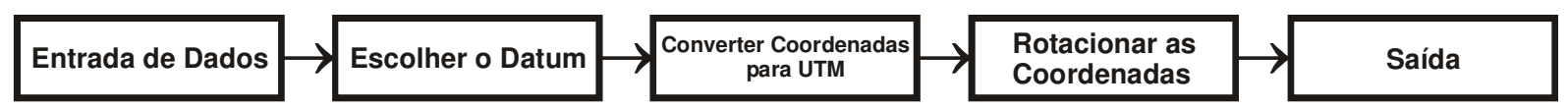

FIGURA 2. Fluxograma das etapas de trabalho da planilha. Flowchart of the steps on the spreadsheet work.

A entrada de dados consiste em fornecer a largura de trabalho da operação agrícola realizada, as coordenadas geográficas dos pontos A e B, e as coordenadas coletadas durante a operação, em que todos esses dados são coletados pelo receptor GNSS RTK. Essas coordenadas são inseridas na planilha em formato de graus decimais, formato geralmente exportado pelos receptores GNSS de mercado, facilitando a entrada de dados. Deve-se atentar para o fato de que os pontos A e B do sistema de orientação a ser avaliado e do receptor GNSS RTK devem ser gerados ao mesmo tempo, em campo.

O segundo passo é escolher o Datum em que foram coletados os dados. A planilha já possui os parâmetros necessários para operar em WGS-84 e SAD-69. Em seguida, as coordenadas são transformadas em coordenadas planas UTM (Universal Transversa de Mercator) de acordo com as equações da Defense Mapping Agency (DMA, 1989). Esta transformação é necessária para se obterem os valores de coordenadas em metros e facilitar o cálculo dos erros. A partir das coordenadas dos pontos A e B, também transformados para UTM, calcula-se o ângulo de inclinação do conjunto de dados em relação a uma linha de referência vertical, conforme ilustrado na Figura 3. 


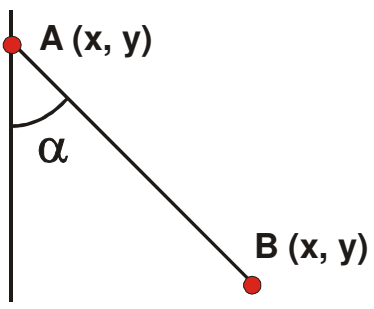

(a)

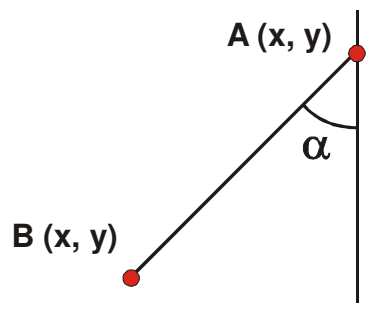

(b)

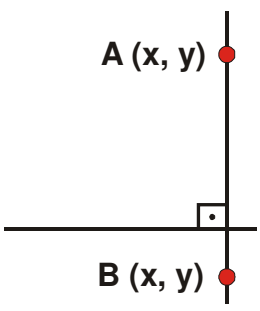

(c)

FIGURA 3. Inclinação da linha de referência A-B de duas formas possíveis (a) e (b) e a linha A-B após rotação das coordenadas (c). Two possible A-B reference line inclinations (a) and (b) and A-B line after coordinates rotation.

Como pode ser observado nas Figuras $3 \mathrm{a}$ e $3 \mathrm{~b}$, existem duas formas de os dados se apresentarem quando inseridos em um Sistema de Informações Geográficas (SIG). Independentemente da maneira em que os dados se apresentam, a planilha calcula o ângulo $\alpha$ pela eq.(1):

$$
\alpha=90-\left[\operatorname{tg}^{-1}\left(\frac{|\mathrm{yA}-\mathrm{yB}|}{|\mathrm{xA}-\mathrm{xB}|}\right)\right]
$$

em que,

$\operatorname{tg}^{-1}$ - tangente inversa;

yA - coordenadas y do ponto A em UTM;

yB - coordenadas y do ponto B em UTM;

$x A$ - coordenadas $x$ do ponto A em UTM, e

$\mathrm{xB}$ - coordenadas $\mathrm{x}$ do ponto $\mathrm{B}$ em UTM.

Como na equação as diferenças entre as coordenadas dos pontos estão em módulo, não importa a posição dos pontos A e B no campo; podem estar como apresentadas na Figura 2 ou invertidas. A partir dessa inclinação, aplicam-se as eqs.(2) a (5) para rotacionar as coordenadas de modo que a coordenada $\mathrm{x}$ dos pontos $\mathrm{A}$ e $\mathrm{B}$ se tornem a mesma, ou seja, a linha A-B passa a estar na orientação norte-sul (Figura 3c). As mesmas equações são aplicadas a todos os pontos coletados em campo.

$$
\begin{aligned}
& \mathrm{x}=\mathrm{x}_{\text {UTM }} \cos \alpha+\mathrm{y}_{\text {UTM }} \operatorname{sen} \alpha \\
& \mathrm{y}=-\mathrm{x}_{\text {UTM }} \operatorname{sen} \alpha+\mathrm{y}_{\text {UTM }} \cos \alpha \\
& \mathrm{x}=-\mathrm{x}_{\text {UTM }} \cos \alpha+\mathrm{y}_{\text {UTM }} \operatorname{sen} \alpha \\
& \mathrm{y}=\mathrm{x}_{\text {UTM }} \operatorname{sen} \alpha+\mathrm{y}_{\text {UTM }} \cos \alpha
\end{aligned}
$$

em que,

$\mathrm{x}$ - coordenadas do eixo $\mathrm{x}$ rotacionadas;

$\mathrm{y}$ - coordenadas do eixo y rotacionadas;

xUTM - coordenadas do eixo x em UTM;

yUTM - coordenadas do eixo y em UTM, e

$\alpha$ - ângulo de inclinação dos dados.

Depois que a linha de referência A-B é colocada na orientação norte-sul, são criadas linhas de referência paralelas à linha $\mathrm{A}-\mathrm{B}$, espaçadas com base na largura de trabalho especificada na entrada dos dados. A posição das linhas de referência será de acordo com as coordenadas dos dados, ou seja, se estão à esquerda ou à direita da linha A-B. As eqs.(2) e (3) são aplicadas quando a inclinação dos dados se apresenta como a Figura 3a, e as eqs.(4) e (5), quando as coordenadas se comportam como a Figura 3b. Se os sistemas de orientação fossem perfeitos, as coordenadas de cada passada do trator seriam exatamente iguais às linhas de referência, portanto o cálculo dos erros 
se baseia simplesmente na diferença no eixo x entre a coordenada de cada ponto coletado em campo com sua respectiva referência. Os dados de saída da planilha separam os resultados automaticamente, de maneira que o conjunto de dados de cada passada fique em uma coluna diferente, com os erros em distâncias métricas. Além disso, já apresentam resultados de média, mínimo, máximo, desvio-padrão e coeficiente de variação de cada passada, sempre considerando erros positivos e negativos, ou seja, se o veículo errar para a esquerda da referência, o valor dos erros é negativo, e se o erro for para a direita, é positivo (Figura 4).

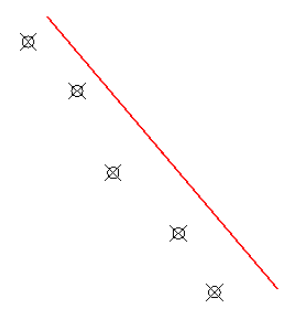

(a)

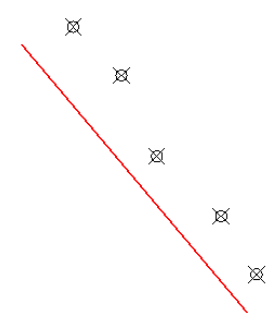

(b)

FIGURA 4. Pontos coletados com erros à esquerda (a) e à direita (b) da linha de referência. Points collected with errors at left (a) and at right (b) of the reference line.

Os erros de trajetórias para o ensaio realizado foram mensurados a partir do desvio perpendicular à reta de referência, como descrito por TAYLOR et al. (2003), explicitado pelas eqs.(6) e (7). A partir do erro médio e desvio-padrão, foram calculadas e apresentadas as frequências acumuladas dos desvios observados.

$$
\begin{aligned}
& \text { ErroRMS }=\sqrt{\frac{1}{n} \sum_{i=1}^{n} d_{i}^{2}} \\
& d_{i}^{2}=\left(x_{i}-x_{t}\right)^{2}+\left(y_{i}-y_{t}\right)^{2}
\end{aligned}
$$

em que,

Erro RMS - média do somatório de todos os desvios registrados, em metros;

$\mathrm{n}$ - número de desvios registrados;

$\mathrm{d}_{\mathrm{i}}$ - desvio, em metros;

$\mathrm{x}_{\mathrm{i}}$ - coordenada $\mathrm{x}$ referência;

$\mathrm{x}_{\mathrm{t}}$ - coordenada $\mathrm{x}$ observada;

$\mathrm{y}_{\mathrm{i}}$ - coordenada y de referência, e

$\mathrm{y}_{\mathrm{t}}$ - coordenada y observada.

A partir dos erros de paralelismo mensurados por meio da planilha eletrônica, foi analisada a frequência acumulada de erro em cada tratamento, e posteriormente os erros com $50 \%$ de probabilidade e com probabilidade $2 \sigma(95,4 \%$ de probabilidade) foram submetidos à análise da variância, pelo teste F de Snedecor, a 5\% de probabilidade, utilizando o pacote estatístico SAS®.

\section{RESULTADOS E DISCUSSÃO}

No gráfico da Figura 5, é apresentada a frequência acumulada dos erros para os dois sistemas de correção utilizados com a barra de luz. Nesta situação, verifica-se que os erros foram menores para o sinal de correção diferencial via satélite de acurácia submétrica em relação ao posicionamento autônomo com auxílio de algoritmo interno. 


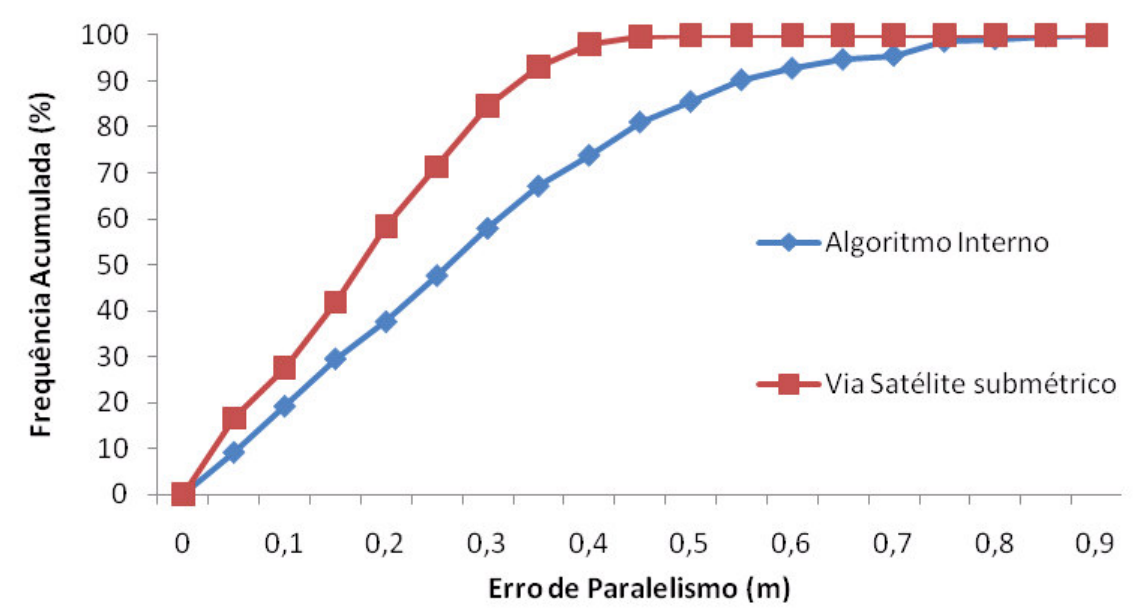

FIGURA 5. Frequência acumulada dos erros (\%) para os sinais de GNSS utilizados com a barra de luz. Accumulated frequency of errors (\%) for the GNSS signals used on the light bar.

No gráfico da Figura 6, é apresentado o desempenho da barra de luz com a frequência acumulada dos erros nos dois sistemas de correção utilizados no sistema automático de orientação. De forma muito semelhante à barra de luz, nesta situação, verifica-se que os erros foram menores para o sinal de correção diferencial via satélite em relação ao posicionamento autônomo com auxílio de algoritmo interno.

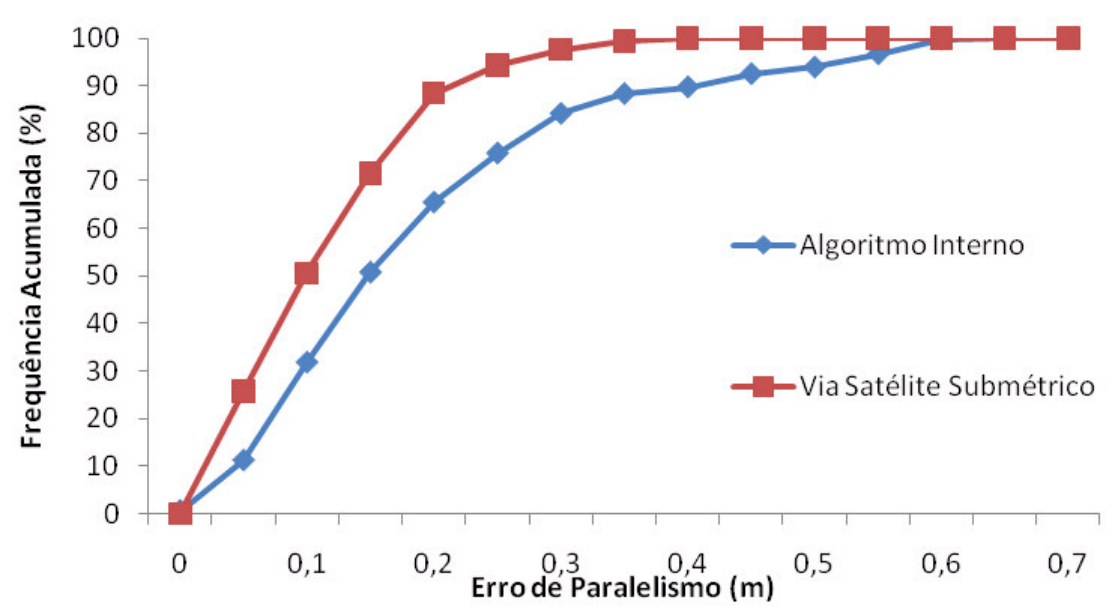

FIGURA 6. Frequência acumulada dos erros (\%) para os sinais de GNSS utilizados com o sistema automático de orientação. Accumulated frequency of errors (\%) for the GNSS signals used on the auto pilot.

Nas Tabelas 1 e 2 apresentam os resultados da análise comparativa realizada para os dois sistemas de correção diferencial para o sinal de GPS utilizados na barra de luz e no sistema automático de orientação, respectivamente. Verifica-se, na Tabela 1 , que o erro médio (50\% de probabilidade), de $0,342 \mathrm{~m}$, foi coerente, de acordo com o especificado pelo seu fabricante, quando utilizando sinal GNSS com auxílio de algoritmo interno. Para o sinal de GPS com correção diferencial via satélite, o erro foi de 0,208 m. Da mesma forma, os desvios com probabilidade de

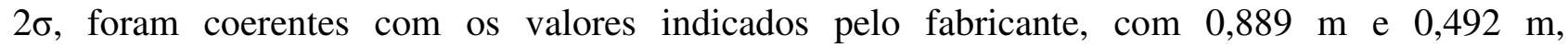
respectivamente. Em ambas as probabilidades, as médias dos dois diferiram significativamente entre si, a $5 \%$ de probabilidade.

Os resultados da Tabela 2 expressam os erros obtidos com o sistema automático de orientação e indicam que o erro médio para o sinal com auxílio de algoritmo interno foi de $0,364 \mathrm{~m}$ e para o 
sinal com correção diferencial via satélite, o erro médio foi de $0,134 \mathrm{~m}$. Da mesma forma, os desvios com probabilidade $2 \sigma$ foram coerentes para esses dois sinais, com $0,628 \mathrm{~m}$ e $0,363 \mathrm{~m}$, respectivamente. Em ambas as probabilidades, as médias dos dois diferiram significativamente entre si a $5 \%$ de probabilidade.

TABELA 1. Análise de variância para as correções de sinal de GPS utilizadas na barra de luz. Analysis of variance for the GPS differential corrections used on the light bar.

\begin{tabular}{ccccc}
\hline Sinal de Correção & \multicolumn{2}{c}{ Erro Médio RMS } & \multicolumn{2}{c}{ Erro 2 $\sigma$} \\
& $(\mathrm{m})$ & $\operatorname{Pr}>\mathrm{F}$ & $(\mathrm{m})$ & $\operatorname{Pr}>\mathrm{F}$ \\
\hline Algoritmo interno & 0,342 & $<0,0001$ & 0,889 & $<0,0001$ \\
Submétrico via satélite & 0,208 & & 0,492 & $<$ \\
\hline
\end{tabular}

TABELA 2. Análise de variância para as correções de sinal de GPS utilizadas com o sistema automático de orientação. Analysis of variance for the GPS differential corrections used on auto pilot.

\begin{tabular}{|c|c|c|c|c|}
\hline \multirow{2}{*}{ Sinal de Correção } & \multicolumn{2}{|c|}{ Erro Médio RMS } & \multicolumn{2}{|c|}{ Erro $2 \sigma$} \\
\hline & $(\mathrm{m})$ & $\operatorname{Pr}>F$ & (m) & $\operatorname{Pr}>F$ \\
\hline $\begin{array}{c}\text { Algoritmo interno } \\
\text { Submétrico via satélite }\end{array}$ & $\begin{array}{l}0,364 \\
0,134\end{array}$ & $<0,0001$ & $\begin{array}{l}0,628 \\
0,363\end{array}$ & $<0,0001$ \\
\hline
\end{tabular}

Verifica-se, na Tabela 2, que para o sistema automático de orientação, em que o sistema não apresenta interferência do operador, os valores são inferiores aos da barra de luz, fato observado nos desvios $2 \sigma$, onde os erros com o sinal via satélite foram menores para o sistema automático de orientação, em relação à barra de luz, com exceção para a média do sinal com auxílio de algoritmo interno.

Os erros apresentados para as correções avaliadas, nos dois equipamentos de sistema de direcionamento utilizados, mostraram-se compatíveis com operações que exijam acurácia de paralelismo da ordem de decímetros, tais como aplicações de insumos sólidos a lanço e pulverizações. As duas tecnologias - barra de luz e sistema automático de orientação - tiveram comportamentos bastante semelhantes em termos de desvios de paralelismo, com destaque para o sistema automático de orientação com sinal de correção via satélite, que apresentou os melhores resultados.

O método utilizado na avaliação da acurácia dos sistemas de orientação e dos sinais de correção supre as necessidades da avaliação em campo. A planilha eletrônica específica, utilizada na rotação das coordenadas e mensuração dos desvios em relação às trajetórias, mostrou-se como instrumento de viabilização do método de avaliação em que se tem um receptor GPS RTK como referência. Com o uso da planilha, foi possível a aquisição e o processamento de grande quantidade de dados, o que contribui para mitigação de possíveis erros do método. Alterações futuras de sua forma de apresentação, mudando o ambiente de planilha para algo mais próximo do usuário, como telas de programas feitos por meio de linguagem de programação de alto nível, serão bem-vindas do ponto de vista estético e, sobretudo, funcional.

\section{CONSIDERAÇÕES FINAIS}

A análise dos dados com o uso de planilha eletrônica permitiu a coleta de grande quantidade de dados, dispensando a necessidade de mensurações em campo, como a utilização de marcação, balizas e trenas, permitindo maior rapidez com menos chances para erros experimentais. A planilha tornou-se uma ferramenta que pode ser utilizada para avaliação de qualquer sistema de orientação, seja barra de luz ou sistema automático de orientação, bastando para isso que os dados de posicionamento real do veículo sejam coletados com um receptor de alta acurácia. 
O sinal de correção via satélite de nível submétrico apresentou valores inferiores de erros operando tanto no sistema automático de orientação quanto na barra de luz. Tanto a barra de luz quanto o sistema automático de orientação tiveram comportamentos semelhantes em termos de erros de paralelismo, com destaque para o sistema automático de orientação com sinal via satélite que apresentou os melhores resultados.

\section{AGRADECIMENTOS}

À FAPESP, pelo apoio à execução do projeto.

\section{REFERÊNCIAS}

ABDINE, A.Z.; HEIDMAN, B.C.; UPADHYAYA, S.K.; HILLS, D.J.; Application of RTK GPS based auto-guidance system in agricultural production. ASAE Paper No. 021152. ASAE, St. Joseph, MI.2002.

ACIAR. AUSTRALIAN CENTER FOR INTERNATIONAL AGRICULTURAL RESEARCH. Project 9209: Conservation tillage and controlled traffic. Canberra: ACIAR, 1998.

ANTUNIASSI, U.R.; BAIO, F.H.R. Orientação de máquinas agrícolas por uma barra de luz (Outback S) utilizando algoritmo para correção do erro GPS. In: CONGRESSO BRASILEIRO DE AGRICULTURA DE PRECISÃO, 1., 2004, Piracicaba. Anais... Piracicaba: ESALQ, 2004. 1 CDROM.

BAIO, F.H.R.; ANTUNIASSI, U.R. Ensaio comparativo da acurácia de um sistema de direcionamento via satélite e por cabo de aço na orientação de máquinas agrícolas. Energia na Agricultura, Botucatu, v.23, p.60-73, 2008.

DEFENCE MAPPING AGENCY-DMA. The Universal Grids: Universal Transverse Mercator and Universal Polar Stereographic, Fairfax 1989. (Technical Manual 8358.2).)

EHSANI, M.R.; SULLIVAN, M.; WALKER, J.T. A Method of evaluating different guidance systems. Chicago: ASAE, 2002. (Paper No. 02-1155).

GAN-MOR, S.; CLARK, R.L. DGPS-based automatic guidance - implementation and economical analysis. Documento: ASAE, 2001. (Paper No. 01-1192).

GAN-MOR, S.; CLARK, R.L.; UPCHURCH, B.L. Implement lateral position accuracy under RTK-GPS tractor guidance. Computers and Electronics in Agriculture, New York, v.59, p.31-38, 2007.

HADAS, A. Controlled traffic in Israel. Acta Horticulture, The Hague, n.210, p.43-67, 1987.

MOLIN, J.P.; CERRI, D.G.P.; BAIO, F.H.R.; TORREZAN, H.F.; ESQUERDO, J.C.D.M.; RÍPOLI, M.L.C. Evaluation of a light bar for parallel swathing under different forward speeds. In: WORLD CONGRESS IN AGRICULTURE AND NATURAL RESOURCES, 2002, Foz do Iguaçu. Proceedings... p.190-195.

POVH, F.P.; SPEKKEN, M.; SALVI, J.V.; MACHADO, T.M.; MOLIN, J.P. Metodologia para análise de paralelismo em sistemas de orientação utilizando planilha eletrônica. In: SIMPÓSIO INTERNACIONAL DE AGRICULTURA DE PRECISÃO, 4., 2007, Viçosa. Anais... Viçosa: Universidade Federal de Viçosa, 2007. 1 CD-ROM.

SILVA, C.B.; OLIVEIRA, T.C.A. de; MOLIN, J.P.; SHIROTA, R. Sistemas de Piloto Automático: Aspectos Técnicos e Econômicos. In: SIMPOSIO INTERNACIONAL DE AGRICULTURA DE PRECISÃO, 4., 2007, Viçosa. Anais... Viçosa: Universidade Federal de Viçosa, 2007. 1 CD-ROM. 